\title{
Re: The outcome of pregnancy in women with cystic fibrosis: a UK population based descriptive study. (First comment on BJOG-20-0094.R1)
}

\author{
Jamie Duckers ${ }^{1}$, Daniella Schlueter ${ }^{2}$, Rhiannon Phillips ${ }^{2}$, Rebecca Cosgriff ${ }^{2}$, Oluwaseun \\ Esan $^{2}$, Shantini Parajothy ${ }^{2}$, Deni Williams², Rachel Norman ${ }^{2}$, David Taylor Robinson ${ }^{3}$, \\ and Siobhan Carr ${ }^{2}$ \\ ${ }^{1}$ Cardiff and Vale University Health Board \\ ${ }^{2}$ Affiliation not available \\ ${ }^{3}$ University of Liverpool
}

October 1, 2020

\section{Dear Editor in Chief}

We read with interest "The outcome of pregnancy in women with cystic fibrosis: a UK population based descriptive study ${ }^{1}$." We thank the authors for this timely paper which highlights the growing need for robust clinical data capture for Cystic Fibrosis maternal and infant health.

This is a rapidly expanding area of interest as the landscape of CF care is changing dramatically, particularly in the era of CFTR modulation. Clinical teams caring for people living with CF are increasingly being asked about pregnancy and potential risks to mothers and their babies. As the authors highlight data on pregnancy in CF historically has mainly been small case series from single sites carried out before CFTR modulators were widely available. The guidelines referenced are somewhat dated as the authors allude to.

The authors describe the strength of the data collection in the form of the UK OSS. However, over the 24 month period only 71 cases are collected. The authors also reference around $30-40$ women with CF being pregnant per year in the UK.* We suspect that there are a significant proportion of missing cases within this study suggested by an interrogation of the UK CF Registry data. The UK CF Registry has been sponsored and hosted by the CF Trust since 2007. CF clinical care teams enter data at every specialist centre and clinic across the UK, with over $99 \%$ of people with CF consenting to their data being submitted. Data from the UK CF Registry over the time period reported by this study revealed 64, 71 and 58 women having babies in 2015, 2016 and 2017 respectively. Although it is not possible to exactly match the dates (March 2015 to February 2017) described within this study to the CF registry data it is likely that there were considerably more than 71 cases in a two year period. **

There remain queries regarding the clinical details of those women reported in this study. Eight of the 71 (11\%) had no underlying CFTR mutation available and $51 \%$ were reported as pancreatic sufficient which is much higher than would be expected from a cohort of adults with CF in the UK. For 15 of the women included in the case series, lung function is not described.

One of the main conclusions of the paper is that gestational age is highly correlated with FEV1. We find the presentation of this misleading as this is most likely the case because women with poorer lung function had pre-term caesareans or had labour induced earlier. Although this doesn't change the correlation it may mean that it is due to the intervention. 
There is an ongoing UK Government funded project, CF Prosper, looking at pregnancies in women with CF using UK CF Registry and US CF Foundation registry data from 2003 to current (https://cfprosper.yolasite.com). We expect to be able to report on over 1000 pregnancies and help to delineate predictive factors for successful pregnancies and examine subsequent maternal disease trajectory after starting a family. Using the largest dataset yet available we also hope to develop a decision-making tool in conjunction with women living with $\mathrm{CF}$ and their clinical care teams to enable women with $\mathrm{CF}$ to make more informed choices regarding pregnancy and starting a family.

Yours faithfully,

Jamie Duckers, Cardiff and Vale University Health Board, UK

Daniela Schlueter, Liverpool University, UK

Rhiannon Phillips, Cardiff Metropolitan University, UK

Rebecca Cosgriff, Cystic Fibrosis Trust, UK

Esan Oluwaseun, Liverpool University, UK

Shantini Paranjothy, Aberdeen University, UK

Deni Williams, Cardiff Metropolitan University, UK

Rachel Norman Cardiff and Vale University Health Board, UK

David Taylor Robinson, Liverpool University, UK

Siobhan Carr, Royal Brompton Hospital, London UK

1) Ashcroft A, Chapman SJ, Mackillop L. The outcome of pregnancy in women with cystic fibrosis: a UK population-based descriptive study. BJOG. 2020 Jul 19. doi: 10.1111/1471-0528.16423. Epub ahead of print. PMID: 32683738.

*There appears to be an error in the referencing numbering within the article as no reference 13 exists

**Details on how to request data from the UK CF Registry are available via www.cysticfibrosis.org.uk/registry 